\title{
LRIG2 promotes the proliferation and cell cycle progression of glioblastoma cells in vitro and in vivo through enhancing PDGFR $\beta$ signaling
}

\author{
QUNGEN XIAO, MINHAI DONG, FANGLING CHENG, FENG MAO, WEIFENG ZONG, KANG WU, \\ HEPING WANG, RUIFAN XIE, BAOFENG WANG, TING LEI and DONGSHENG GUO \\ Department of Neurosurgery, Tongji Hospital, Tongji Medical College, \\ Huazhong University of Science and Technology, Wuhan, Hubei 430030, P.R. China
}

Received March 13, 2018; Accepted June 29, 2018

DOI: $10.3892 /$ ijo.2018.4482

\begin{abstract}
The leucine-rich repeats and immunoglobulin-like domains (LRIG) gene family, comprising LRIG1, 2 and 3, encodes integral membrane proteins. It has been well established that LRIG1 negatively regulates multiple growth factor signaling pathways and is considered to be a tumor suppressor; however, the biological functions of LRIG2 remain largely unexplored. It was previously demonstrated that LRIG2 positively regulates epidermal growth factor receptor (EGFR) signaling, the most common aberrant receptor tyrosine kinase (RTK) signaling in glioblastoma multiforme (GBM), which promotes GBM growth. In the present study, the effect of LRIG2 on the proliferation of GBM cells was further addressed, as well as the possible mechanisms underlying the regulatory effect of LRIG2 on platelet-derived growth factor receptor $\beta$ (PDGFR $\beta$ ) signaling, another common oncogenic RTK signaling pathway in GBM. First, the expression levels of endogenous LRIG2 and PDGFR $\beta$ were found to vary notably in human GBM, and the LRIG2 expression level was positively correlated with the expression level of PDGFR $\beta$. Furthermore, to the best of our knowledge, this is the first study to demonstrate that LRIG2 promoted the PDGF-BB-induced proliferation of GBM cells in vitro and in vivo through regulating the PDGFR $\beta$
\end{abstract}

Correspondence to: Dr Baofeng Wang, Department of Neurosurgery, Tongji Hospital, Tongji Medical College, Huazhong University of Science and Technology, 1095 Jiefang Avenue, Wuhan, Hubei 430030, P.R. China

E-mail:wbf620@163.com

Abbreviations: GMB, glioblastoma multiforme; LRIG, leucine-rich repeats and immunoglobulin-like domains; RTK, receptor tyrosine kinase; PDGFR, platelet-derived growth factor receptor; Stat3, signal transducer and activator of transcription 3

Key words: leucine-rich repeats and immunoglobulin-like domains, platelet-derived growth factor receptor $\beta$, glioblastoma, proliferation, cell cycle signaling-mediated cell cycle progression. Mechanistically, LRIG2 has the ability to physically interact with PDGFR $\beta$, promoting the total expression and the activation of PDGFR $\beta$, and enhancing its downstream signaling pathways of Akt and signal transducer and activator of transcription 3 and the effectors of key regulators of cell cycle progression, resulting in increased GBM cell proliferation. Collectively, these data indicated that LRIG2 may serve as a tumor promoter gene in gliomagenesis by positively regulating PDGFR $\beta$ signaling, another important oncogenic RTK signaling pathway, in addition to the previously reported EGFR signaling in GBM modulated by LRIG2, and validated LRIG2 as a promising therapeutic target for the treatment of GBM characterized by multiple aberrant RTK signaling.

\section{Introduction}

Glioblastoma multiforme (GBM) is the most common primary malignant brain tumor in adults. Despite treatment optimization and improved standard of care, the median survival of patients with GBM is only 14.5-16.6 months (1). Thus, the development of novel therapeutic targets is crucial for the future treatment of GBM.

Over the last decade, substantial efforts have been focused on elucidating the molecular characteristics of GBM, in order to identify molecular subsets as targets for specific targeted therapies, potentially improving the outcome (2). The most common genetic characteristics of GBM are activated oncogenic receptor tyrosine kinase (RTK) signaling pathways via epidermal growth factor receptor (EGFR) or platelet-derived growth factor receptor (PDGFR), and inactivated tumor suppressor pathways regulated by retinoblastoma protein and tumor protein p53 (3). Among these, PDGFR signaling plays a key role in the pathogenesis of GBM and represents a target for tyrosine kinase inhibitors (4), whereas PDGFR inhibitors to date have failed to elicit significant responses in patients with GBM. Due to the key role of PDGFR activation in GBM progression and the yet obscure mechanisms underlying the poor clinical outcomes with PDGFR inhibitors, the understanding of the endogenous regulators of PDGFR signaling has been an area of intensive research. 
The leucine-rich repeats and immunoglobulin-like domains (LRIG) gene family is composed of three paralogs, namely LRIG1, LRIG2 and LRIG3, which encode a family of integral membrane proteins, with a signal peptide, an extracellular part consisting of 15 leucine-rich repeats (LRR) and three immunoglobulin-like domains, followed by a transmembrane domain and a cytoplasmic tail (5). LRIG proteins have attracted attention as key regulators of growth factor receptors, including receptor tyrosine and serine/threonine kinases (6). LRIG1, the most extensively investigated LRIG family member, is considered to be a tumor suppressor by negatively regulating the signaling pathways mediated by ErbB (7), MET (8) and RET (9) receptor tyrosine kinases. LRIG1 is downregulated and associated with a favorable prognosis in various types of cancer $(6,10-15)$, including glioma $(16,17)$; however, our knowledge on the biological and molecular functions of mammalian LRIG2 in tumors is limited. It has been reported that LRIG2 expression is associated with poor survival in oligodendroglioma (18) and uterine cervical carcinoma (19), and wild-type mice developed PDGFB-induced gliomas at a higher frequency and of higher malignancy compared with LRIG2-deficient mice (20). Over the past decade, our research team has focused on the functions of LRIGs in gliomagenesis, and demonstrated that LRIG2 promotes the growth of GBM by positively modulating EGFR-mediated signaling $(21,22)$, which adds to the evidence supporting the hypothesis that LRIG2 may play an oncogenic role in the progression of glioma, possibly contrary to the role of LRIG1 $(23,24)$; however, more compelling evidence is required to support the concept of LRIG2 as a tumor promoter in GBM and to elucidate the mechanistic insights.

In the present study, the role of LRIG2 in the progression of GBM and the possible underlying mechanisms by which LRIG2 modulates PDGFR $\beta$ signaling were investigated. First, the association between the expression levels of LRIG2 and PDGFR $\beta$ in human GBM was evaluated. Furthermore, stable GBM cells with LRIG2 overexpression or knockdown were established and the effects of LRIG2 on PDGF-BB-induced proliferation and cell cycle progression were investigated in vitro and in vivo. Finally, the mechanism underlying these effects was explored. To the best of our knowledge, the present study is the first to investigate the effect of LRIG2 on PDGFR $\beta$ signaling pathways, and thereby on the proliferation and cell cycle progression of human GBM.

\section{Materials and methods}

Cell culture. The U87 human GBM cell line was purchased from American Type Culture Collection (ATCC; Manassas, VA, USA). U87 cells were cultured in Dulbecco's modified Eagle's medium (DMEM) containing 10\% fetal bovine serum (FBS) in a humidified incubator with $5 \% \mathrm{CO}_{2}$ at $37^{\circ} \mathrm{C}$. According to Allen et al (25), the fact that U87 from ATCC originated from an unknown patient and is not the original U87 established at the University of Uppsala does not affect the authenticity of U87 as a human GBM cell line. Thus, the use of U87 from ATCC in the present study is considered appropriate and the results from the use of U87 as a GBM cell line are not affected.
shRNA-mediated gene knockdown. To knock down LRIG2 expression, a vector-based short hairpin RNA (shRNA) expression system was used. A total of two nucleotide sequences, targeting LRIG2 (NM_014813) nucleotides 451-471 (shRNA1) and 1379-1399 (shRNA2), and one non-silencing scrambled shRNA (scr) were designed and synthesized (Table I). The shRNA inserts were digested with EcoRI and AgeI prior to ligation into the pLKO.1-TRC cloning vector (Addgene Inc., Cambridge, MA, USA) according to the manufacturer's protocol. The constructed plasmid contained the ampicillin resistance gene for selection of ampicillin-resistant colonies in bacteria. All the inserted sequences were verified by DNA sequencing.

Stable cell transduction. The stable LRIG2-overexpressing U87 cells (U87-LRIG2) and the corresponding control cells (U87-Con) had been established and used previously (22). U87 cells were stably infected with lentivirus expressing pLKO.1-TRC-scr or pLKO.1-TRC-LRIG2shRNA using Lenti-X lentiviral Expression Systems (Clontech Laboratories, Inc., Mountainview, CA, USA) according to the manufacturer's instructions as described previously (22). In brief, 1 day before the transfection, $4 \times 10^{6} 293 \mathrm{~T}$ cells (ATCC) were seeded in a 100-mm plate and cultured in DMEM containing 10\% Tc-free FBS (Clontech Laboratories, Inc.). A total of $3 \mu \mathrm{g}$ of each plasmid DNA, including the constructed pLKO.1-TRC-scr and pLKO.1-TRC-LRIG2sh, was mixed with Lenti-X HT Packaging Mix (Clontech Laboratories, Inc.) and added to the $293 \mathrm{~T}$ cell culture medium. After $48 \mathrm{~h}$ of incubation, the lentivirus-containing supernatants were harvested and filtered through a $0.45-\mu \mathrm{m}$ filter (EMD Millipore, Billerica, MA, USA). The Lenti-X qRT-PCR Titration Kit (Clontech Laboratories, Inc.) was used to determine the viral titer. U87 cells were seeded into 6-well plates at a density of $3 \times 10^{5}$ cells/well and incubated overnight prior to transduction. The viral supernatant was mixed with culture medium containing $4 \mu \mathrm{g} / \mathrm{ml}$ polybrene (Sigma-Aldrich; Merck KGaA, Darmstadt, Germany), added to the cells and transduced for $24 \mathrm{~h}$. Then, the transduced cells were selected with culture medium containing $1 \mu \mathrm{g} / \mathrm{ml}$ puromycin (Sigma-Aldrich; Merck KGaA) for 2 weeks and the puro-resistant clones that represented possible stably transduced cells were expanded for further experiments.

Patients and tissue samples. A total of 40 GBM samples were obtained by surgical resection from Tongji Hospital, Tongji Medical College, Huazhong University of Science and Technology (Wuhan, China), between 2012 and 2013. The subset of patients with GBM included 24 men and 16 women aged 38-76 years (median age, 58 years). All the patients provided written informed consent for the use of their tissues, and the study was approved by the Institutional Review Board of Tongji Hospital (ID: 20121202). Following surgical resection, fresh tumors were immediately fixed with formalin and embedded in paraffin for routine histopathological evaluation and immunohistochemical staining.

Immunohistochemical staining. The immunohistochemical staining procedures were performed as previously described (22). Briefly, GBM samples from humans and nude mice were fixed in 
a phosphate-buffered $4 \%$ formaldehyde solution and embedded into paraffin blocks using standard methods. Tissue sections $(4-\mu \mathrm{m})$ were labeled with the following primary antibodies at the indicated concentrations: Anti-LRIG2 rabbit polyclonal antibody 1:100 (cat. no. ab157452; Abcam, Cambridge, UK), anti-PDGFR $\beta$ rabbit monoclonal antibody 1:100 (cat. no. 3169), anti-pPDGFR $\beta$ rabbit monoclonal antibody 1:50 (cat. no. 4549), anti-pAkt rabbit monoclonal antibody 1:100 (cat. no. 4058), anti-pStat3 rabbit monoclonal antibody 1:100 (cat. no. 9145) (all from Cell Signaling Technology, Inc., Danvers, MA, USA), anti-cyclin B1 rabbit polyclonal antibody 1:100 (cat. no. BA0766), anti-Cyclin D1 rabbit polyclonal antibody 1:100 (cat. no. BA0770) (both from Boster Biological Technology), anti-Ki67 rabbit monoclonal antibody 1:100 (cat. no. ab16667), anti-PCNA rabbit monoclonal antibody 1:100 (cat. no. ab92552;) (both from Abcam). The evaluation of the immunostaining was performed by two independent observers in a blinded manner. In brief, positive immunohistochemical staining in tumor cells was evaluated with respect to the intensity of positive staining and the percentage of positive tumor cells. From these scoring data, a four-grade semiquantitative score was defined as follows: 0 , no or very faint immunoreactivity (IR) or immunopositive cells $<5 \%$; 1 , weak IR or immunopositive cells $5-20 \%$; 2 , moderate IR or immunopositive cells $26-50 \%$; 3 , strong IR or immunopositive cells $>50 \%$. A total score based on low intensity and fraction (scored as 0 or 1 ) vs. high intensity and fraction (scored as 2 or 3 ) was created for each patient and statistically analyzed.

Immunofluorescence. Cells were fixed with $4 \%$ paraformaldehyde for $1 \mathrm{~h}$ at $4^{\circ} \mathrm{C}$, permeabilized with $0.25 \%$ Triton X-100 for 5 min, blocked with $10 \%$ bovine serum albumin (Boster Biological Technology) for $1 \mathrm{~h}$ and labeled with primary anti-LRIG2 1:50 (cat. no. ab157452; Abcam), anti-PDGFR $\beta$ 1:100 (cat. no. 3169; Cell Signaling Technology, Inc.), and anti-Flag 1:100 (cat. no. F1804; Sigma-Aldrich; Merck KGaA) antibodies overnight at $4^{\circ} \mathrm{C}$, as previously described (22). Following washing with phosphate-buffered saline (PBS), cells were incubated with fluorescein isothiocyanate or cyanine-labeled secondary antibodies (1:100; ProteinTech Group, Inc., Chicago, IL, USA) and subjected to immunofluorescence microscopy using appropriate filters.

Cell proliferation assay. In order to examine cellular proliferation, cell counting kit-8 (CCK8) assays and cell count analysis were performed. For CCK 8 assays, $5 \times 10^{3}$ cells in $200 \mu 1$ suspension per well were seeded in triplicate in 96-well flat-bottomed plates and allowed to attach overnight at $37^{\circ} \mathrm{C}$. Subsequently, the cells were treated with indicated concentrations of PDGF-BB for indicated times. At the indicated time points, CCK8 (CK04; Dojindo Molecular Technologies, Inc., Kumamoto, Japan) was added to the wells prior to incubation for $2 \mathrm{~h}$ at $37^{\circ} \mathrm{C}$. The optical density was measured with a microplate reader (Thermo Fisher Scientific, Inc., Waltham, MA, USA) using a wavelength of $450 \mathrm{~nm}$. For cell count determination, U87 GBM cells with LRIG2 overexpression or downregulation and the corresponding control cells were seeded in triplicate at a density of $10 \times 10^{4}$ cells/well in 6-well plates. Similar to the CCK8 assay, cells were allowed to attach, followed by serum starvation with DMEM for $24 \mathrm{~h}$.
Table I. Oligonucleotide sequences of LRIG2-specific siRNA.

\begin{tabular}{llc}
\hline Name & $\begin{array}{c}\text { siRNA } \\
\text { sequences } \\
\left(5^{\prime} \otimes 3^{\prime}\right)\end{array}$ & $\begin{array}{c}\text { Target } \\
\text { sequence } \\
\text { on LRIG2 } \\
\text { cDNA }\end{array}$ \\
\hline shRNA1 & AATCGGTTGTCTAACTGGAAC & $451-471$ \\
shRNA2 & AAGGAAACCAGATTAAGTCAA & $1379-1399$ \\
Scramble & CAACAAGATGAAGAGCACCAA & \\
\hline
\end{tabular}

LRIG2, leucine-rich repeats and immunoglobulin-like domain 2.

Synchronized cells were then treated with or without STI571 (cat. no. 13139; Cayman Chemical Company, Ann Arbor, MI, USA) $10 \mu \mathrm{M}$ for $2 \mathrm{~h}$ prior to stimulation with PDGF-BB (cat. no. 100-14B; PeproTech, Rocky Hill, NJ, USA) $50 \mathrm{ng} / \mathrm{ml}$ for $24 \mathrm{~h}$. At the end of the experiment, the cells were enzymatically detached, and the cell numbers were counted in triplicate for each group.

Reverse transcription-quantitative polymerase chain reaction (RT-qPCR). Synchronized cells were cultured with DMEM containing $10 \%$ FBS for $48 \mathrm{~h}$. Total RNA was isolated and quantitative RT-qPCR was performed as previously described (24). The DNA primer sequences of LRIG2 were designed as follows: Sense, 5'-CAGTGCATAGCTGGAGGGA GTC-3' and antisense, 5'-TACAATGATGAGAAGCTGATTG GCTGCA-3'. The primer sequences of the control GAPDH were designed as follows: Sense, 5'-CACCAGGGCTGCTTTTA ACTCTGGTA-3' and antisense, 5'-CCTTGACGGTGCCAT GGAATTTGC-3'.

Flow cytometric analysis of the cell cycle. The cells were synchronized to the G0/G1 stage by $24 \mathrm{~h}$ of serum deprivation and then cultured in DMEM with $0.5 \%$ FBS with or without PDGF-BB for an additional $24 \mathrm{~h}$. Cell cycle analysis was performed by propidium iodide (PI) staining and flow cytometry. In brief, cells were collected and washed twice with cold PBS and resuspended in pre-cooled $70 \%$ ethanol at $4{ }^{\circ} \mathrm{C}$ overnight. Subsequently, fixed cells were collected by centrifugation and incubated with RNase $(25 \mu \mathrm{g} / \mathrm{ml})$ and PI $(50 \mu \mathrm{g} / \mathrm{ml})$ at $37^{\circ} \mathrm{C}$ for $30 \mathrm{~min}$ in the dark. The number of cells in the G0/G1, S and G2/M phases was analyzed by flow cytometry using a FACSCalibur Flow Cytometer (BD Biosciences, Franklin Lakes, NJ, USA).

Soft agar colony formation assay. Anchorage-independent cell proliferation was determined by soft agar colony formation assay as previously described (22). Briefly, 6-well plates were first covered with a bottom layer of $0.6 \%$ agar made in $10 \%$ FBS DMEM and $4 \times 10^{4}$ cells per well were embedded in triplicate into $0.3 \%$ top agar gel containing DMEM supplemented with $10 \%$ FBS. Cells were fed with $0.5 \%$ FBS DMEM with or without PDGF-BB (50 ng/ml) every third day and allowed to grow for 2 weeks. For each well, 30 randomly selected fields were photographed under a microscope (ECLIPSE TS100; Nikon, Tokyo, Japan) with a x10 microscopic lens and a x6 optical lens (magnification, x60). The colonies were then 
counted and the mean number of colonies per field was calculated. Each experiment was performed in triplicate.

In vivo tumor formation in nude mice. All animal procedures were conducted in accordance with the guidelines for animal experimentation, and the animal protocol used in this study was approved by the Institutional Review Board of Tongji Hospital, Tongji Medical College, Huazhong University of Science and Technology (IRB ID: 2011A01). A total of 10 immune-deficient female nude mice (BALB/c), aged 6 weeks and weighing 20-22 g, were purchased from the Wuhan Laboratory Animal Center, bred at the facility of laboratory animals at Tongji Medical College, and randomly assigned to two groups $(n=5$ per group): U87-scr and U87-LRIG2sh. For subcutaneous inoculation, a total of $2 \times 10^{6}$ cells in $100 \mu 1$ PBS were injected subcutaneously into the right flank of each mouse as previously described (22). Tumor volume was measured with calipers every 5 days and calculated using the formula: Volu me $=$ length $\mathrm{x}$ width $^{2} \mathrm{x}(\pi / 6)$ (length, larger diameter in $\mathrm{mm}$; width, smaller diameter in $\mathrm{mm}$ ). At the end of the experiment, the mice were euthanized and the tumors were surgically harvested, measured, weighed, fixed in $4 \%$ paraformaldehyde overnight and analyzed by immunohistochemistry.

Western blotting and co-immunoprecipitation. Western blotting and co-immunoprecipitation were performed as previously described $(22,24)$. The primary antibodies used in western blotting at the indicated concentrations were as follows: Anti-pPDGFR $\beta$ rabbit monoclonal antibody 1:500 (cat. no. 4549), anti-PDGFR $\beta$ rabbit monoclonal antibody 1:500 (cat. no. 3169), anti-pAkt rabbit monoclonal antibody 1:1,000 (cat. no. 4058), anti-Akt rabbit polyclonal antibody (cat. no. 9273), anti-pStat3 rabbit monoclonal antibody 1:1,000 (cat. no. 9145), anti-Stat3 rabbit monoclonal antibody 1:1,000 (cat. no. 4904) (all from Cell Signaling Technology, Inc.), anti-cyclin B1 rabbit polyclonal antibody 1:500 (cat. no. BA0766), anti-cyclin D1 rabbit polyclonal antibody 1:500 (cat. no. BA0770), $\beta$-actin mouse monoclonal antibody 1:1,000 (cat. no. BM0627) (all from Boster Biological Technology). Co-immunoprecipitation of LRIG2 and PDGFR $\beta$ were performed using flag-tagged LRIG2-overexpressing U87 cells. Briefly, cells were washed twice with ice-cold PBS and lysed with IP lysis buffer (cat. no. 87787; Thermo Fisher Scientific, Inc.). Lysates were pre-cleared by adding $20 \mu \mathrm{l}$ of Protein A/G Agarose (cat. no. A10001; Abmart, Berkeley Heights, NJ, USA), followed by overnight incubation with anti-Flag (cat. no. F1804; Sigma-Aldrich; Merck KGaA) or anti-PDGFR $\beta$ (cat. no. 3169; Cell Signaling Technology, Inc.) on a rocker at $4^{\circ} \mathrm{C}$. Immunocomplexes were captured by incubating with Protein A/G Agarose for $2 \mathrm{~h}$ at $4^{\circ} \mathrm{C}$, followed by washing with ice-cold lysis buffer to eliminate non-specific interactions. The agarose-bound immunocomplexes were then eluted by boiling the beads in 2X SDS loading buffer (cat. no. P0015B; Beyotime Institute of Biotechnology, Shanghai, China) for $10 \mathrm{~min}$ at $100^{\circ} \mathrm{C}$. The whole-cell lysates were used as positive controls. In negative controls, primary antibodies used for co-IP were replaced with corresponding isotype $\mathrm{IgG}$ controls. Eluted proteins and the input (whole lysates) were subjected to western blotting.

Statistical analysis. Statistical analyses were performed using SPSS 16.0 software for Windows (SPSS Inc., Chicago, IL,
USA). The significance of the correlation between LRIG2 and PDGFR $\beta$ in the immunohistochemical staining analysis was determined using Pearson's $\chi^{2}$ test. Multiple comparisons were performed using one-way analysis of variance followed by Bonferroni as a post-hoc test. Statistical differences between the two groups were analyzed with the Student's t-test. Values are expressed as the mean \pm standard deviation of at least three independent experiments. $\mathrm{P}<0.05$ was considered to indicate a statistically significant difference.

\section{Results}

Protein expression level of LRIG2 is positively correlated with the level of PDGFR $\beta$ in human GBM. To investigate the correlation between the expression levels of LRIG2 and PDGFR $\beta$ in human GBM, the protein expression levels of LRIG2 and PDGFR $\beta$ were determined in 40 patient-derived biopsies of GBM, obtained from surgical resections using immunohistochemical staining. According to the total score based on the relative intensity of LRIG2 expression, the patients were categorized into two groups: LRIG2 high expression (scored as 2 or 3) and LRIG2 low expression (scored as 0 or 1). Of the 20 cases in the LRIG2 high expression group, the PDGFR $\beta$ IR of 7 cases was defined as low expression and of 13 cases as high expression. Of the 20 cases in the LIRG2 low expression group, the PDGFR $\beta$ IR of 14 cases was scored as low expression and of 6 cases as high expression. The difference in PDGFR $\beta$ IR was significant between the LRIG2 high expression and low expression groups $(\mathrm{P}<0.05$; Fig. 1 and Table II), which indicated that the protein expression level of PDGFR $\beta$ was higher in GBMs with high LRIG2 expression, compared with GBMs with low LRIG2 expression.

Establishment of the stable LRIG2 knockdown GBM cell line. In order to evaluate the role of LRIG2 in GBM, the expression of LRIG2 was downregulated via shRNA mediated-knockdown in U87 cells. A total of three plasmids expressing two nucleotide sequences targeting LRIG2 (shRNA1 and shRNA2) and one non-silencing scramble shRNA (scr) (Table I) were constructed and stably transduced into U87 GBM cells. RT-qPCR and western blotting were used to verify the mRNA and protein expression levels of LRIG2, respectively, in stably transduced cells. Compared with the scramble control cells, LRIG2 transcripts were markedly reduced in the shRNA1and shRNA2-transduced cells (Fig. 2A). In line with the RT-qPCR results, LRIG2 protein levels were also significantly decreased in the shRNA1- and shRNA2-transduced cells (Fig. 2B). These results indicated that the expression levels of LRIG2 were notably downregulated by LRIG2 shRNAs, and the transduced cells with shRNA1, which exhibited higher knockdown efficiency, were selected for the subsequent experiments. Furthermore, fluorescence microscopy was used to investigate the expression of LRIG2 and PDGFR $\beta$ in U87 cells with LRIG2 knockdown by shRNA1. The results demonstrated that the fluorescent intensity of LRIG2 was markedly attenuated in the U87-LRIG2sh cells (Fig. 2C). Notably, the fluorescent intensity of PDGFR $\beta$ was also significantly decreased (Fig. 2C), similar to the result of LRIG2 expression being positively correlated with the expression of PDGFR $\beta$ in human GBM samples. 
Table II. Protein expression levels of LRIG2 and PDGFR $\beta$ in human glioblastoma tissues.

\begin{tabular}{lrr}
\hline & \multicolumn{2}{c}{ PDGFR $\beta$ expression } \\
\cline { 2 - 3 } LRIG2 expression & High & Low \\
\hline High & 13 & 7 \\
Low & 6 & 14 \\
\hline $\begin{array}{l}\text { 'High' or 'Low' indicates the protein expression level based on the } \\
\text { total score of immunohistochemical staining. LRIG2, leucine-rich } \\
\text { repeats and immunoglobulin-like } \\
\text { derived growth factor receptor } \beta .\end{array}$ \\
\hline
\end{tabular}

LRIG2 promotes PDGF-BB-stimulated proliferation of human GBM cells in vitro. To determine the potential effects of the LRIG2 protein on PDGF-BB-induced cell proliferation of GBM cells, U87 cells were first treated with different concentrations of PDGF-BB for various durations. The CCK8 assay was used to evaluate cell proliferation. Overexpression of LRIG2 was found to markedly promote the PDGF-BB-induced proliferation of U87 GBM cells in a concentration- and time-dependent manner (Fig. 3A and B). Congruently, downregulation of LRIG2 markedly inhibited the PDGF-BB-induced proliferation of U87 cells in a concentration- and time-dependent manner (Fig. 3C and D). Subsequently, we investigated the effects of LRIG2 on the PDGF-BB-induced anchorage-independent growth of U87 cells, a property that mimics tumorigenesis in vivo. Using soft agar colony formation assays, it was observed that, when stimulated with PDGF-BB, LRIG2-overexpressing GBM cells formed more colonies compared with the control cells (Fig. 4A); however, when LRIG2 was downregulated in U87 cells, the effect of LRIG2 on enhancement of PDGF-BB-induced anchorage-independent cell growth was abrogated (Fig. 4B), indicating that the LRIG2 protein enhances the ability of PDGF-BB-stimulated anchorage-independent proliferation in GBM cells.

Furthermore, STI571, the most promising PDGFR $\beta$ inhibitor, was used to investigate whether the impact of LRIG2 on the proliferation of GBM cells relies on PDGFR $\beta$ RTK regulation. U87 GBM cells with LRIG2 overexpression or downregulation and the corresponding control cells were pretreated with or without STI571 to block PDGFR $\beta$ signaling, and then stimulated with or without PDGF-BB for $24 \mathrm{~h}$. As shown in Fig. 3E and F, pretreatment of LRIG2-overexpressing U87 cells with STI571 resulted in marked attenuation of the increased proliferation induced by PDGF-BB; however, when LRIG2 was downregulated, the PDGF-BB-induced proliferation of GBM cells was markedly decreased, and pretreatment with STI571 exerted no significant effect on PDGF-BB-induced proliferation. Taken together, these findings confirmed that the LRIG2 protein promotes PDGF-BB-induced proliferation of human GBM cells in vitro by regulating the activation of PDGFR $\beta$.

Effects of LRIG2 on the PDGF-BB-stimulated cell cycle distribution of GBM cells. To investigate the mechanism underlying

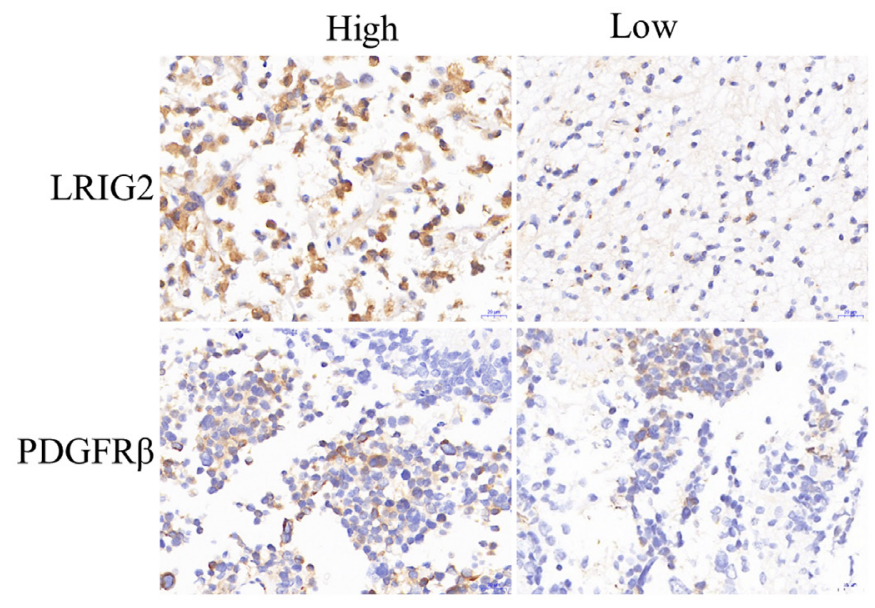

Figure 1. LRIG2 expression levels positively correlates with the expression levels of PDGFR $\beta$ in primary glioblastoma tissues. Representative images of immunohistochemical staining of glioblastoma specimens for LRIG2 and PDGFR $\beta$ in high-expressing cases and low-expressing cases, respectively (scale bar, $20 \mu \mathrm{m}$ ). LRIG2, leucine-rich repeats and immunoglobulin-like domain 2; PDGFR $\beta$, platelet-derived growth factor receptor $\beta$.

LRIG2 promoting the proliferation of PDGF-BB-induced GBM cells, an experiment was performed to assess the effects of LRIG2 on U87 cell cycle progression stimulated by PDGF-BB. The synchronized cells were harvested, cultured in DMEM with $0.5 \%$ FBS with or without PDGF-BB for $24 \mathrm{~h}$, and the cell cycle distribution was analyzed by flow cytometry. The results revealed that the percentage of cells in the G0/G1 phase was markedly decreased and the percentage of cells in the $\mathrm{S}$ or $\mathrm{G} 2 / \mathrm{M}$ phase was markedly increased in the PDGF-BB-induced LRIG2-overexpressing U87 cells compared with the control cells (Fig. 5A). Concordantly, downregulation of LRIG2 caused increased accumulation of cells in the G0/G1 phase and a significantly decreased percentage of cells in the $\mathrm{S}$ or $\mathrm{G} 2 / \mathrm{M}$ phase (Fig. 5B), which was in line with the results reported previously (21). More importantly, when stimulated with PDGF-BB, LRIG2-knockdown GBM cells exhibited markedly increased accumulation in the G0/G1 phase and a strikingly decreased percentage of cells in the $\mathrm{S}$ or $\mathrm{G} 2 / \mathrm{M}$ phase compared with the scramble control cells (Fig. 5B). Taken together, these results demonstrated that the LRIG2 protein promoted PDGF-BB-induced DNA synthesis and the G0/G1 to $S$ phase cell cycle transition in GBM cells, resulting in a higher number of cells entering the $\mathrm{G} 2 / \mathrm{M}$ phase.

LRIG2 promotes the growth of U87 tumor xenograft through regulating the PDGFR $\beta$ signaling pathway in vivo. The aforementioned data confirmed the role of LRIG2 in promoting the proliferation of human GBM cells in vitro; therefore, the present study was further extended in vivo to investigate the effects of LRIG2 knockdown on U87 GBM xenografts, and to explore the possible underlying mechanisms. As mentioned above, stably transduced U87 GBM cells expressing LRIG2 shRNA or scramble RNA were inoculated via subcutaneous injection to the right flank of mice with severe combined immunodeficiency (SCID). Tumor volume was recorded every 5 days from day 15 post-implantation, and the tumor growth curve results demonstrated that tumor growth was markedly 
A

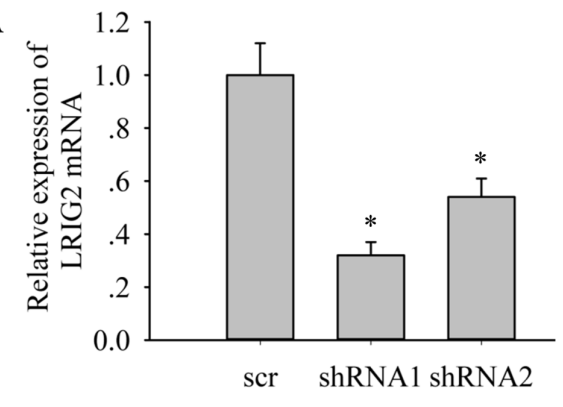

C DAPI

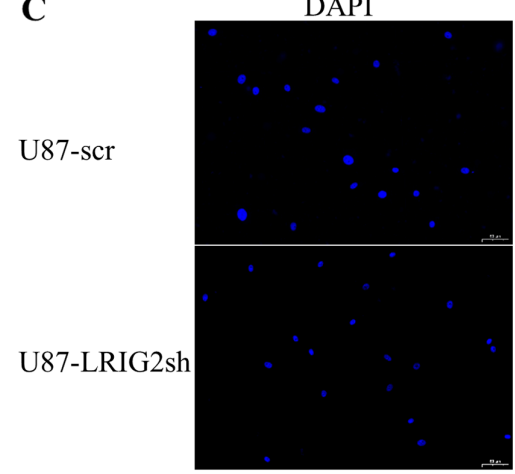

DAPI

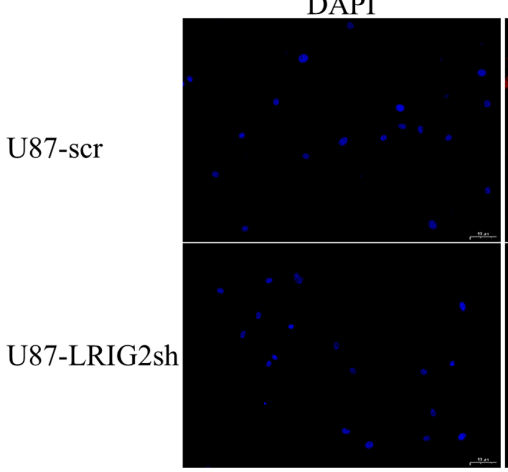

B

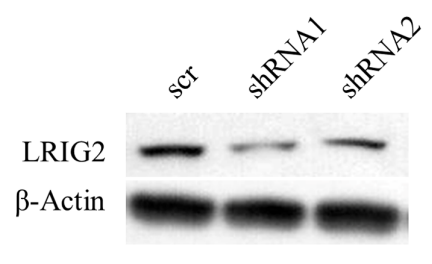

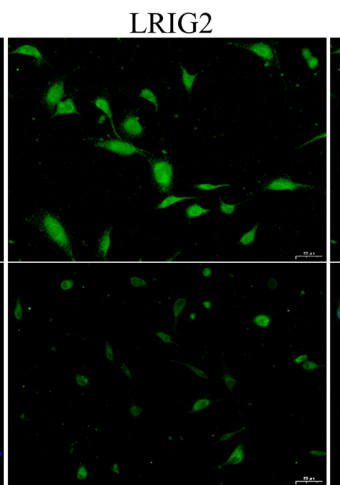

PDGFR $\beta$

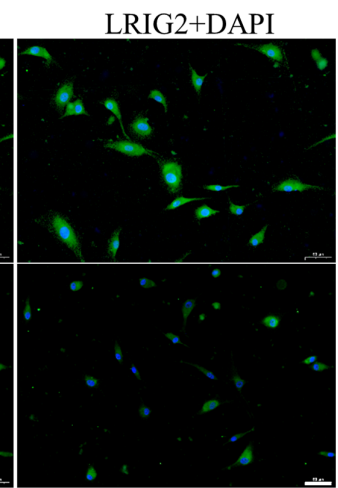

PDGFR $\beta+$ DAPI

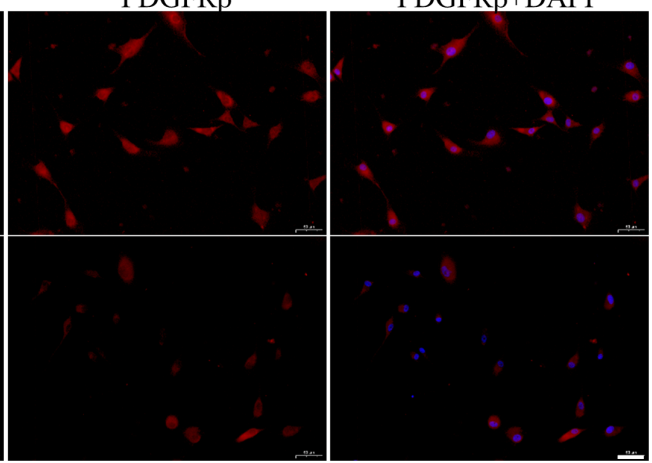

Figure 2. Establishment of stable U87 glioblastoma cells with LRIG2 knockdown, and the effects of LRIG2 downregulation on the expression level of PDGFR $\beta$. U87 cells were transduced with two LRIG2 shRNAs and one control scr, as indicated. (A) The LRIG2 mRNA expression levels were assessed by reverse transcription-quantitative polymerase chain reaction and (B) the LRIG2 protein levels were examined by western blotting ("P<0.05 vs. scr). (C) Stably transduced cells were subjected to immunofluorescence staining for LRIG2 (green) or PDGFR $\beta$ (red), and counterstained with DAPI to show the nuclei (blue). Representative images of three independent experiments are depicted (scale bar, $50 \mu \mathrm{m}$ ). LRIG2, leucine-rich repeats and immunoglobulin-like domain 2; PDGFR $\beta$, platelet-derived growth factor receptor $\beta$; shRNA, short hairpin RNA; scr, scrambled shRNA.

inhibited in mice injected with LRIG2-knockdown U87 cells compared with the scramble group (Fig. 6A and B). At the end of the experimental period (day 40), the xenografts were surgically removed, weighed and subjected to immunohistochemical staining for Ki-67 and proliferating cell nuclear antigen (PCNA), two classical markers of cell proliferation. Consistently, the results revealed that tumor weight was also notably lower in tumors with LRIG2 downregulation (Fig. 6B), and the expression levels of Ki-67 and PCNA were both significantly decreased in LRIG2-shRNA-expressing U87 xenograft tumor tissues (Fig. 6C). Combining the effects of LRIG2 knockdown on tumor growth of in vivo, which were in line with the in vitro data, with the previously reported results that overexpression of LRIG2 promoted the growth of GBM xenograft in vivo (22), the evidence supporting a role of LRIG2 in promoting the proliferation of human GBM cells in vivo is compelling.

Based on the aforementioned in vitro results that LRIG2 promoted the PDGF-BB-induced proliferation of GBM cells via regulating the cell cycle distribution, the possible PDGFR $\beta$ signaling pathways mediated by LRIG2 was further explored in vivo. Fresh LRIG2-overexpressing and LRIG2-silenced tumor xenografts were subjected to immunohistochemical staining for LRIG2, PDGFR $\beta$ and its downstream pathways and effectors. Notably, the expression levels of phosphorylated and total PDGFR $\beta$, as well as its downstream targets, phosphorylated Akt (pAkt) and phosphorylated Stat3 (pStat3), were significantly upregulated in LRIG2-overexpressing GBM xenografts compared with the control xenografts (Fig. 7), whereas the levels of those proteins were markedly decreased in LRIG2-downregulated tumor xenografts (Fig. 7). Furthermore, the expression levels of PDGFR $\beta$ downstream effectors cyclin D1 and cyclin B1, a key regulator of the G1/S phase transition and a $\mathrm{G} 2 / \mathrm{M}$ regulator, respectively, were positively correlated with the expression levels of the LRIG2 protein in GBM xenografts (Fig. 7), providing a convincing explanation for the effects of LRIG2 on the PDGF-BB-stimulated cell cycle distribution of GBM cells in vitro. Collectively, these results 
A
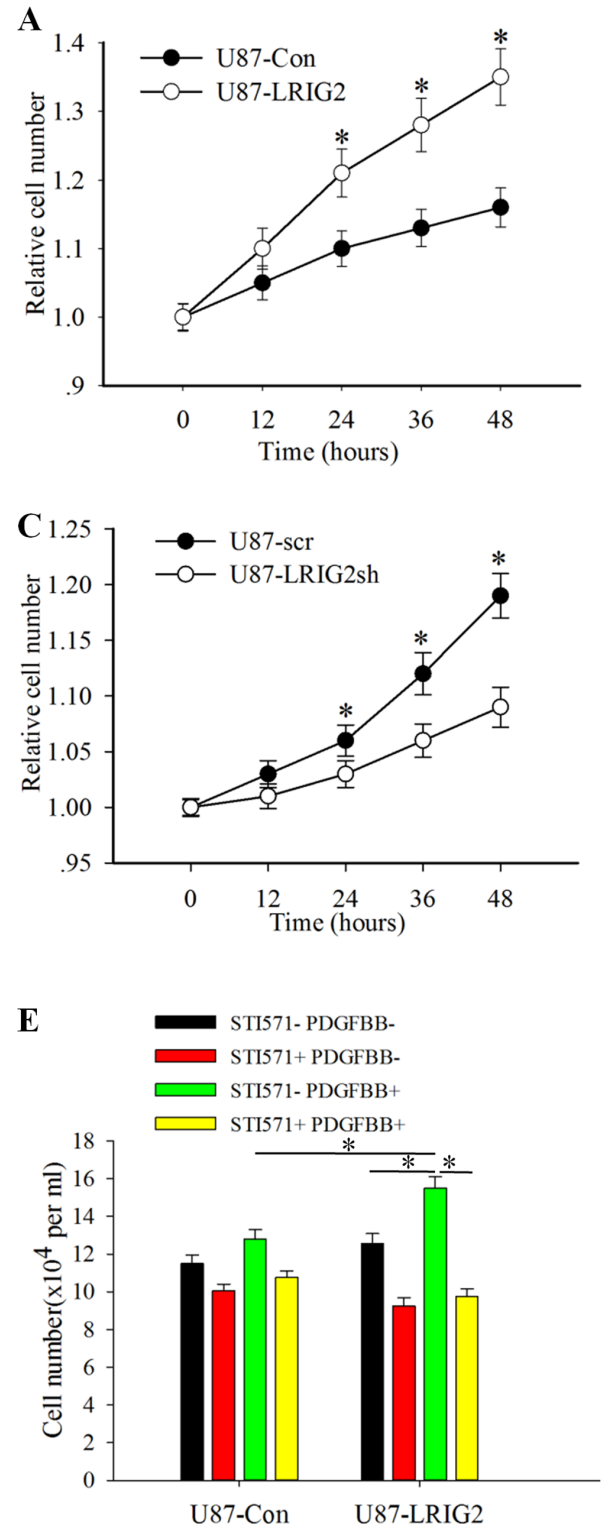
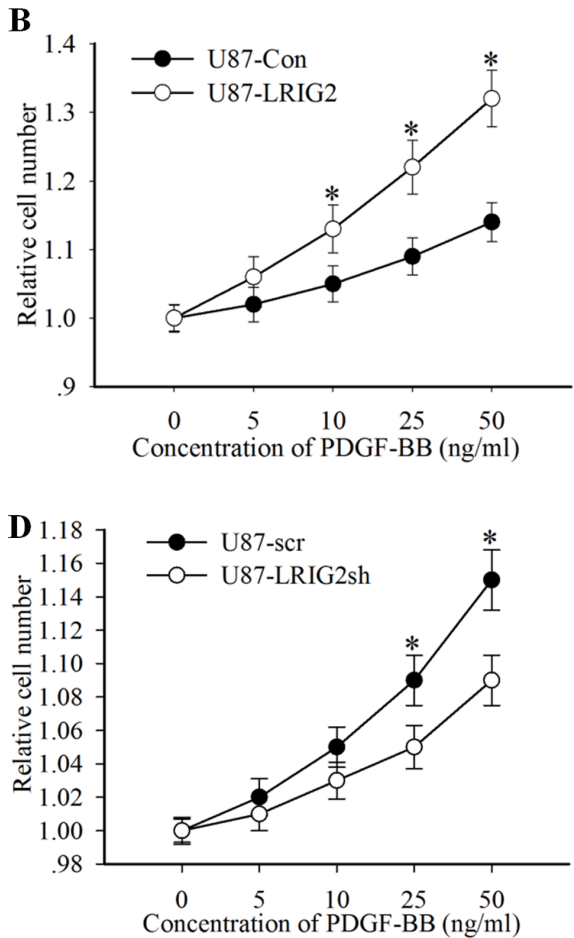

STI571- PDGFBB-
STI571+ PDGFBB-
STI571- PDGFBB+
STI571+ PDGFBB+

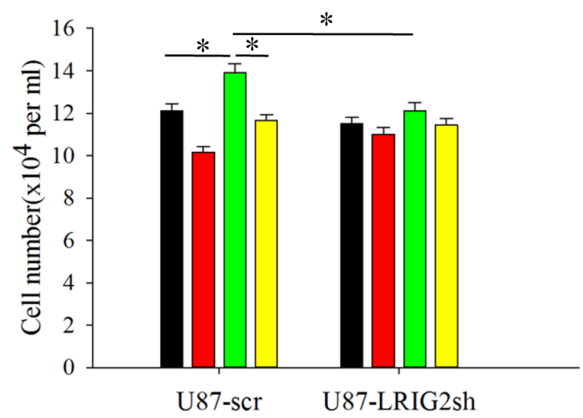

Figure 3. Effects of LRIG2 on PDGF-BB-stimulated proliferation of U87 glioblastoma cells. (A-D) LRIG2 promoted PDGF-BB-induced proliferation of glioblastoma cells in a concentration- and time-dependent manner. U87 cells with LRIG2 overexpression or downregulation were pre-cultured in serum-free medium for $24 \mathrm{~h}$, and then treated with PDGF-BB $(50 \mathrm{ng} / \mathrm{ml})$ for different lengths of time, as indicated, or with various concentrations of PDGF-BB for $48 \mathrm{~h}$. The relative cell numbers were measured by cell counting kit- 8 assay at the times indicated. Points represent mean \pm SD) of at least three independent experiments. ( $\mathrm{P}<0.05$ ). (E and F) Pretreatment of glioblastoma cells with the PDGFR $\beta$ inhibitor STI571 attenuated the LRIG2-regulated proliferation induced by PDGF-BB. Synchronized cells were treated with or without STI571 (10 $\mu \mathrm{M})$ for $2 \mathrm{~h}$ prior to stimulation with PDGF (50 ng/ml) for 24 h. At the end of the stimulation, cells were subjected to a cell counting assay. Data are presented as mean $\pm \mathrm{SD}$ of three independent experiments ( $\mathrm{P}<0.05$ ). SD, standard deviation; LRIG2, leucine-rich repeats and immunoglobulin-like domain 2; PDGFR $\beta$, platelet-derived growth factor receptor $\beta$.

demonstrated that LRIG2 played a pivotal role in promoting the growth of GBM xenografts through regulating PDGFR $\beta$ signaling and its downstream effectors in vivo.

LRIG2 physically interacts with PDGFR $\beta$ RTK in GBM cells. Having demonstrated that LRIG2 promotes the growth of GBM and the possible role of LRIG2 in the enhancement of PDGFR $\beta$ signaling activation, in order to gain insight into the molecular mechanisms underlying the activation of PDGFR $\beta$ by LRIG2, confocal microscopy was performed to evaluate the expression pattern of the two fluorescent proteins in U87 cells. In line with previous studies regarding fluorescent LRIG2 subcellular distribution $(17,26)$, the present study demonstrated via immunofluorescence staining that Flag-tagged LRIG2 was primarily localized in the cytoplasm, and it is noteworthy that the distribution of PDGFR $\beta$ fluorescent signals mirrored the LRIG2 distribution (Fig. 8A), indicating a colocalization of LRIG2 and PDGFR $\beta$ expression in GBM cells. Based on the co-expression pattern and the similar protein structure of LRIG2 and PDGFR $\beta$, both containing extracellular immunoglobulin loops and an intracellular tail (27), it is reasonable to hypothesize that LRIG2 may physically interact with PDGFR $\beta$ in GBM cells. To confirm this hypothesis, the Flag-tagged LRIG2 was precipitated from the cell lysates of LRIG2-overexpressing cells using an anti-Flag antibody. Coprecipitation of PDGFR $\beta$ was observed by probing the resulting blot with anti-PDGFR $\beta$, and when PDGFR $\beta$ was immunoprecipitated from the cell lysates, coprecipitation 

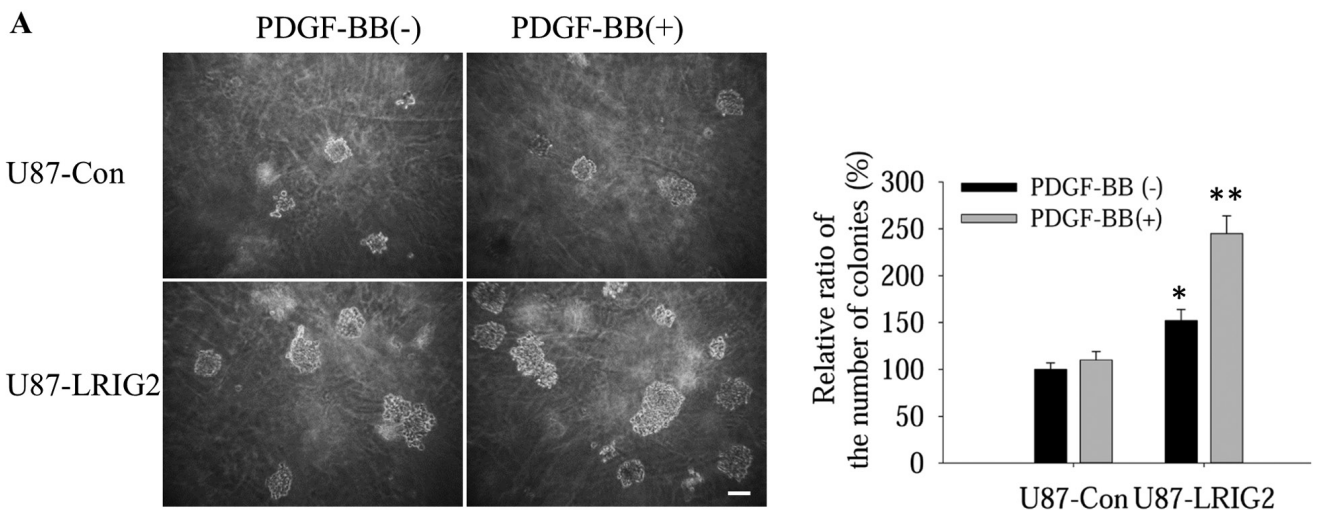

B
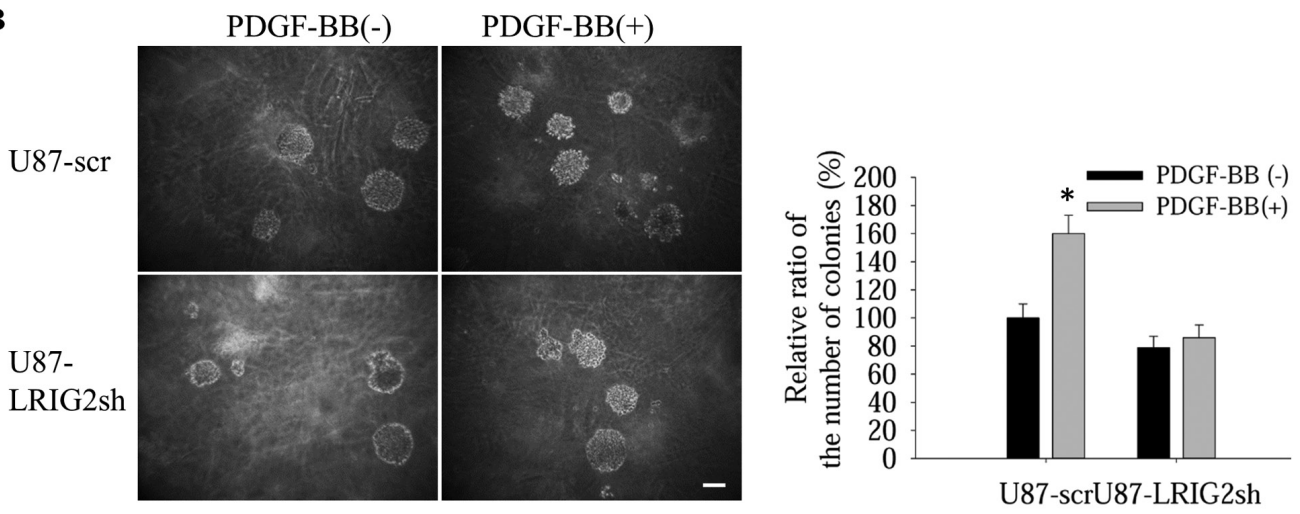

Figure 4. LRIG2 enhances the ability of PDGF-BB-stimulated anchorage-independent growth of U87 glioblastoma cells. Soft agar colony formation assay was used to detect the PDGF-BB-induced anchorage-independent proliferation of the U87 glioblastoma cells with (A) LRIG2 overexpression or (B) LRIG2 knockdown. The representative high-resolution images are depicted (scale bars, $100 \mu \mathrm{m}$ ). Data were normalized by setting the number of colonies in the U87-Con or U87-Scr cells without stimulation of PDGF-BB at 100\% and the relative ratio of the number of colonies (\%) is depicted in the right panel $\left({ }^{*} \mathrm{P}<0.05,{ }^{* *} \mathrm{P}<0.01\right.$ vs. con or scr). All experiments were performed in triplicate with consistent and repeatable results. LRIG2, leucine-rich repeats and immunoglobulin-like domain 2; PDGF, platelet-derived growth factor; con, control; scr, scrambled short hairpin RNA.

of the Flag-LRIG2 was also detected by western blotting with the anti-Flag antibody (Fig. 8B), indicating that LRIG2 physically associates with PDGFR $\beta$. In conclusion, the results presented in Fig. 8 indicate that LRIG2 physically interacts with the PDGFR $\beta$ RTK in GBM cells, which may result in the enhancement of PDGFR $\beta$ signaling activation.

LRIG2 promotes PDGF-BB-induced activation of PDGFR $\beta$ $R T K$ and the downstream Akt and Stat3 signaling pathways and effectors. Subsequently, the physical association of LRIG2 and PDGFR $\beta$ prompted an investigation into whether LRIG2 can modulate the ligand-dependent activation of the PDGFR $\beta$ receptor and its downstream signaling. Stably transduced U87 cells with LRIG2 overexpression or LRIG2 knockdown were synchronized for $24 \mathrm{~h}$ and then treated with or without various concentrations of PDGF-BB for a further $24 \mathrm{~h}$. As depicted in Fig. 9A, the western blotting results demonstrated that the total expression level and the phosphorylation of PDGFR $\beta$ following stimulation with PDGF-BB (the primary activator of PDGFR $\beta$ ) were significantly increased in LRIG2-overexpressing cells and drastically reduced in LRIG2-knockdown cells, as compared with the corresponding control cells. Consistently, the levels of PDGF-BB-induced phosphorylation of Akt and Stat3, two key downstream signaling pathways of PDGFR $\beta$, were markedly increased in LRIG2-overexpressing cells, resulting in substantial accumulation of PDGF-BB-induced downstream effectors of cyclin B1 and cyclin D1, which are key regulators of cell cycle progression (Fig. 9A). Notably, when LRIG2 was downregulated in GBM cells, the PDGF-BB-induced activation of Akt, Stat3 and the downstream effectors of cyclin B1 and cyclin D1 was markedly attenuated (Fig. 9A). To further substantiate the role of LRIG2 in the regulation of PDGFR $\beta$ signaling pathways, STI571, the most promising PDGFR $\beta$ inhibitor, was used to inhibit the phosphorylation of PDGFR $\beta$ prior to detecting the aforementioned downstream pathways. Cells were serum-starved for $24 \mathrm{~h}$ and then treated with STI571 for $2 \mathrm{~h}$ prior to a 10 -min exposure to PDGF-BB, and the total cell lysates were subjected to western blotting. As expected, the expression level of total PDGFR $\beta$ was increased and the activation of PDGFR $\beta$, as well as its downstream targets Akt and Stat3, was significantly enhanced following a 10-min stimulation with PDGF-BB in LRIG2-overexpressing cells, whereas the PDGFR $\beta$ expression level was decreased and the activation of PDGFR $\beta$, Akt and Stat 3 was attenuated in cells with LRIG2 knockdown (Fig. 9B). Notably, the effects of LRIG2 overexpression or knockdown on PDGF-BB-induced enhanced or attenuated activation of Akt and Stat 3 were significantly abrogated following the inhibition of PDGFR $\beta$ phosphorylation by STI571 (Fig. 9B), which demonstrated that LRIG2 played a pivotal role in the regulation of PDGF-BB-induced PDGFR $\beta$ activation and its downstream pathways. Taken together, the aforementioned results provide compelling evidence supporting that LRIG2 


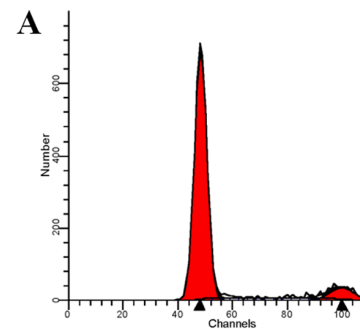

U87-Con

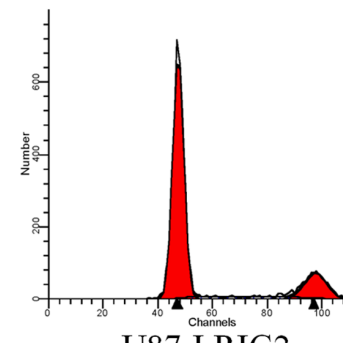

U87-LRIG2

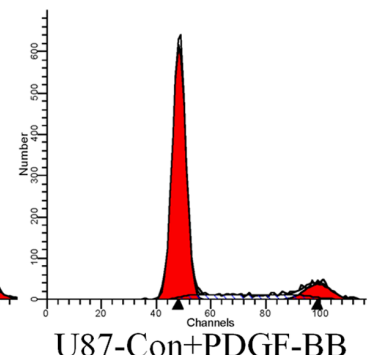

U87-Con+PDGF-BB

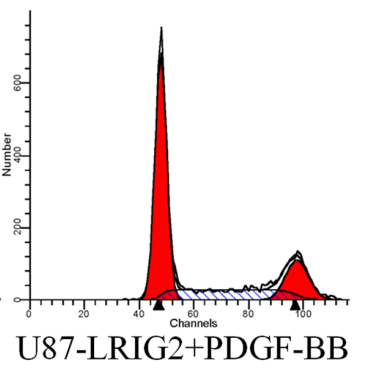

B

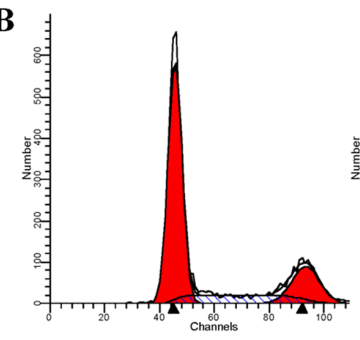

U87-scr

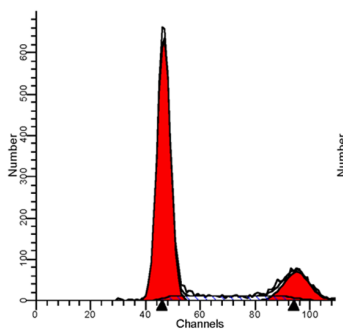

U87-LRIG2sh

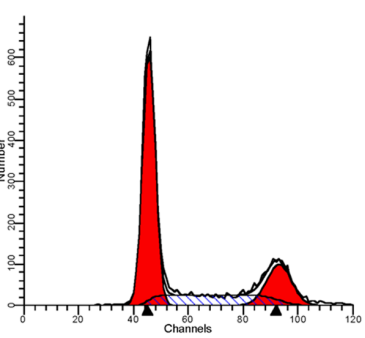

U87-scr+PDGF-BB

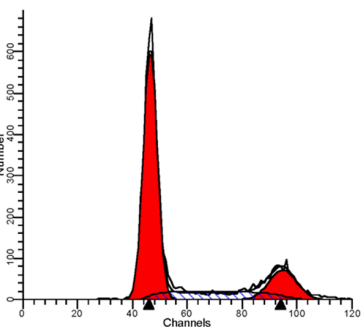

U87-LRIG2sh+PDGFBB
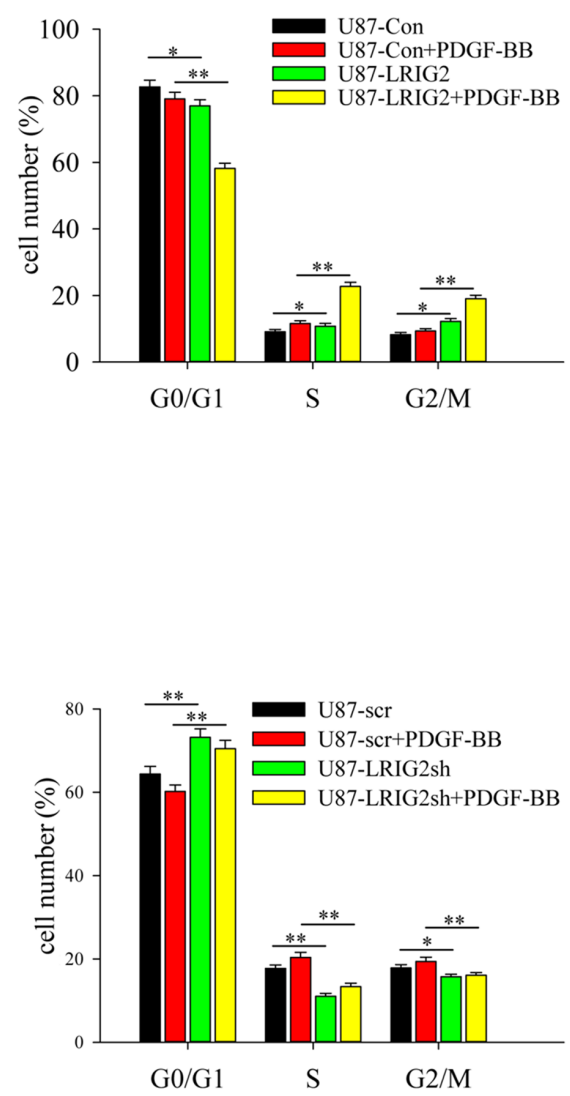

Figure 5. Effects of LRIG2 on PDGF-BB-induced cell cycle distribution. Synchronized U87 glioblastoma cells with (A) LRIG2 overexpression or (B) LRIG2 knockdown were treated with or without PDGF-BB $(50 \mathrm{ng} / \mathrm{ml})$ for $24 \mathrm{~h}$, then stained with propidium iodide and analyzed for cell cycle distribution by using flow cytometry. Three independent experiments were performed and a representative plot is displayed. The percentage of cells in the G0/G1, S and G2/M phases was quantified and plotted. Data are expressed as the mean \pm standard deviation of three independent experiments ( $\left(\mathrm{P}<0.05,{ }^{* * *} \mathrm{P}<0.01\right)$. LRIG2, leucine-rich repeats and immunoglobulin-like domain 2; PDGF, platelet-derived growth factor.

physically interacts with PDGFR $\beta$, leading to stabilization and activation of PDGFR $\beta$ and enhancing the downstream Akt and Stat 3 pathways, ultimately resulting in accumulation of the downstream pro-proliferative effectors of cell cycle proteins to promote the proliferation of GBM cells.

\section{Discussion}

In the present study, it was demonstrated that LRIG2, the least investigated and documented member of the LRIG gene family, is positively correlated with the expression of PDGFR $\beta$ RTK in human GBM, and markedly promoted the PDGF-BB-induced growth of GBM cells in vitro and in vivo through modulating PDGFR $\beta$ activation and its downstream signaling pathways and effectors of cell cycle progression. These data indicated that LRIG2 exerts its pro-tumor effects on GBM cells by positively regulating PDGFR $\beta$ signaling, another important aberrant oncogenic RTK signaling modulated by LRIG2 in GBM, similar to our previously reported EGFR signaling (22).

GBM, the most common and lethal type of malignant primary brain tumor (1), is a devastating and intractable disease with a poor outcome. A hallmark of malignant glioma is activation of aberrant RTK signaling pathways, most commonly caused by EGFR amplification/mutation or PDGFR amplification/overexpression $(28,29)$, the genomic alterations of which ultimately lead to enhanced tumor cell proliferation, increased transition through the cell cycle and resistance to treatment. PDGFRs are well-characterized RTKs in GBM that have been found to be overexpressed in human gliomas of all grades, are most highly expressed in GBM, and are closely associated with GBM initiation and progression $(20,28)$. Similar to previous studies (30), we observed that overexpression of PDGFR $\beta$ was a frequent event 
A

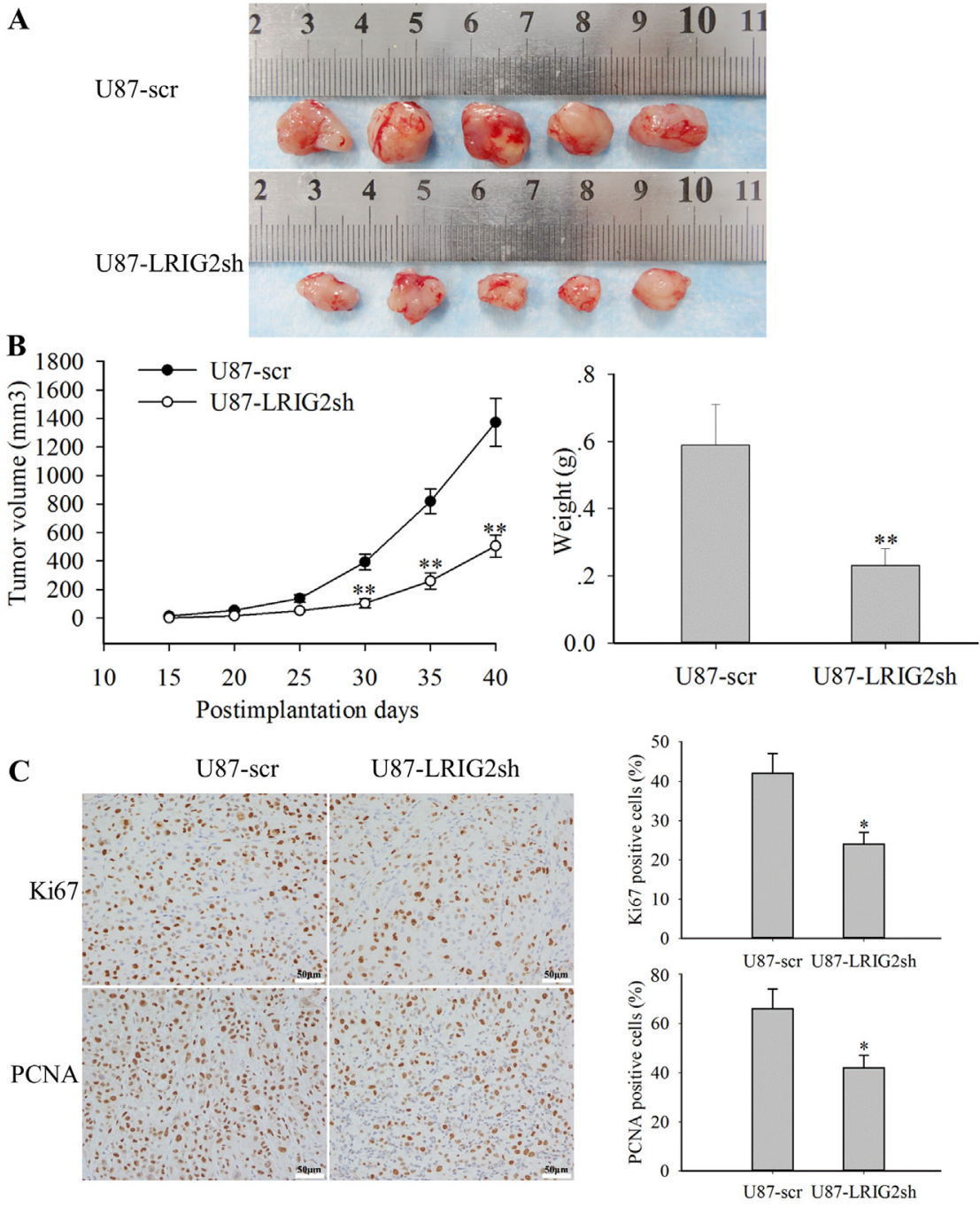

Figure 6. LRIG2 knockdown suppresses the growth of U87 glioblastoma xenografts in vivo. U87 glioblastoma cells stably expressing LRIG2 shRNA or scramble RNA were injected subcutaneously to the flanks of sever combined immunodeficient mice (5 per group). (A) At the end of animal experiment, tumor xenografts were surgically removed, measured and photographed as indicated. (B) Tumor volumes were monitored every 5 days for a total of 40 days from day 15 post-implantation and the growth curves of xenografts were depicted in the left panel graph, and the tumor weights of isolated xenografts at day 40 were displayed in the right panel. Data are shown as the mean \pm standard deviation $\left({ }^{* *} \mathrm{P}<0.01\right)$. (C) Fresh isolated tumor xenografts were fixed in $4 \%$ paraformaldehyde and subjected to immunohistochemical staining for Ki-67 and PCNA. The representative images are depicted in the left panel (scale bars, $50 \mu \mathrm{m}$ ) and the percentages of immunopositive cells of Ki-67 and PCNA in the two groups are depicted in the right panel ("P<0.05). LRIG2, leucine-rich repeats and immunoglobulin-like domain 2; shRNA, short hairpin RNA; PCNA, proliferating cell nuclear antigen.

in human GBM. To the best of our knowledge, this is the first study to demonstrate that the expression levels of PDGFR $\beta$ vary and are positively correlated with the expression levels of LRIG2 in human GBM, indicating that the inter-individual variation in PDGFR $\beta$ expression may be determined by the expression pattern of LRIG2, and PDGFR $\beta$ expression may be positively regulated by LRIG2 in human GBM, which was demonstrated in vitro and in vivo in the present study. Congruently, Rondahl et al (20) used animal models of PDGFB-induced glioma to demonstrate that Lrig2E12-/mice, generated by the ablation of Lrig 2 exon 12, developed lower-grade tumors $(77 \%)$ or had no detectable tumors $(23 \%)$, and Lrig2 $\mathrm{E} 12^{+/+}$mice developed lower-grade tumors $(82 \%)$ or high-grade GBM-like tumors (18\%). The Lrig2E12 $2^{+/}$mice developed PDGFB-driven gliomas at a higher frequency and of higher malignancy compared with Lrig2E12 $2^{-/}$mice (20), suggesting a key promoting role of Lrig2 in the regulation of PDGF signaling and in the development and/or progression of glioma, particularly in mice, consistently with the findings of the present study, which demonstrated that LRIG2 positively regulates PDGFR $\beta$ signaling in human GBM. Furthermore, the present results, which indicated that LRIG2 is positively correlated with PDGFR $\beta$ in human GBM, are also in line with those of a previous study reporting that LRIG2 expression is associated with poor survival of patients with oligodendroglioma, in which dysregulated PDGFR signaling is a common feature (18). To the best of our knowledge, this is the first study demonstrating a positive association between LRIG2 and PDGFR signaling in human GBM, providing compelling evidence in support of the hypothesis that LRIG2 serves as a tumor promoter in human glioma, which is distinct from the functions of LRIG1 $(23,24)$ and LRIG3 in glioma (6).

Furthermore, in order to provide further evidence to support the concept that LRIG2 acts as a tumor promoter by positively regulating PDGFR $\beta$ signaling in GBM, PDGF-BB-induced biological effects and signaling events were analyzed in GBM 


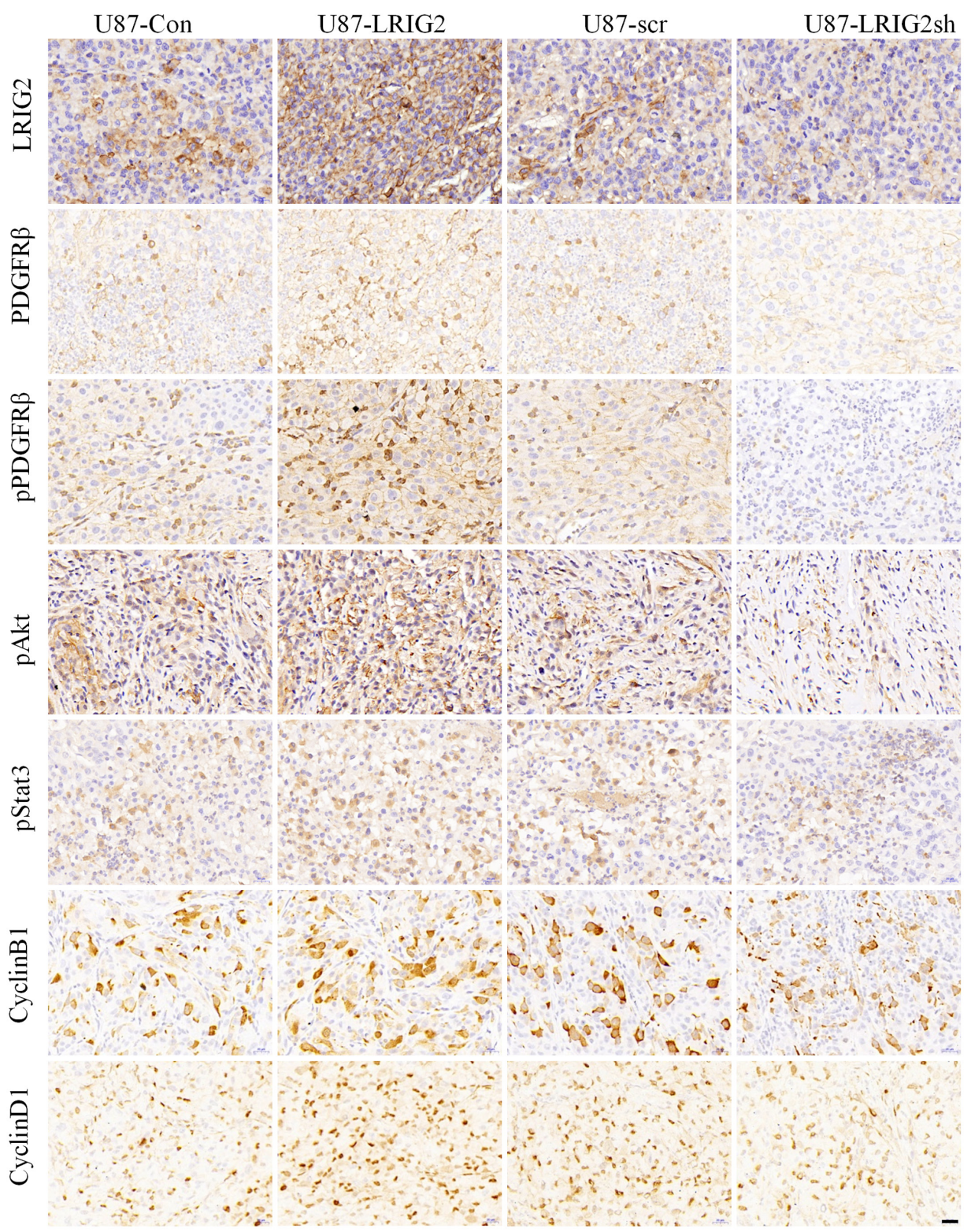

Figure 7. LRIG2 enhances the activation of PDGFR $\beta$ and regulates its downstream pathways and effectors in vivo. A total of four groups of indicated stable U87 glioblastoma cells (U87-LRIG2, LRIG2-overexpressing; U87-Con, control U87 cells; U87-LRIG2sh, LRIG2-knockdown cells; and U87-scr, scramble U87 cells) were injected subcutaneously into the flanks of mice with severe combined immunodeficiency. At the end of the animal experiments, tumor xenografts were isolated and immunohistochemistry was performed to evaluate the expression of LRIG2, PDGFR $\beta$ and its downstream pathways, as indicated. Representative images are depicted (scale bars, $20 \mu \mathrm{m}$ ). LRIG2, leucine-rich repeats and immunoglobulin-like domain 2; PDGFR $\beta$, platelet-derived growth factor receptor $\beta$.

cells with LRIG2 overexpression or downregulation. The pathogenesis of GBM is complex, due to a highly deregulated tumor genome with a network of interconnected signaling pathways of three steps: i) An input step in which membrane receptors are triggered from the signals outside the cell; ii) a core system in which protein kinases transmit the signal to the nucleus; and iii) an output step in which transcription factors regulate the genes that affect various cellular functions (29). Congruently, LRIG2 initially plays a critical role in the first input step by promoting the PDGF-BB-induced activation of PDGFR $\beta$, followed by enhancing the activation of Akt kinases and the Stat 3 transcription factor, the core system of the second step, ultimately resulting in the output step, in which the pro-proliferative cell cycle proteins are accumulated and the uncontrolled proliferation of GBM cells is promoted. Due to the modulation of PDGFR $\beta$ signaling by LRIG2 upstream and the importance of abnormal PDGFR $\beta$ signaling in gliomagenesis, it is reasonable to propose that LRIG2 fits the role of a single critical oncogene, the antagonism of which should abrogate the important downstream deregulated signaling cascades and interrupt cell proliferation. It is well documented that deregulation of the phosphoinositide 3-kinase/Akt pathway is an obligate event frequently observed in gliomagenesis (2), with Akt activation reported in $~ 80 \%$ of human GBMs (31). Stat3 is also aberrantly activated in human GBM tissues and serves as a 'molecular hub' connecting extracellular signals to the transcriptional control of critical cellular events in gliomagenesis, including cell cycle progression, proliferation, angiogenesis and immune evasion (32). Of note, our findings demonstrated that LRIG2 positively regulated 
A

DAPI

Flag

PDGFR $\beta$
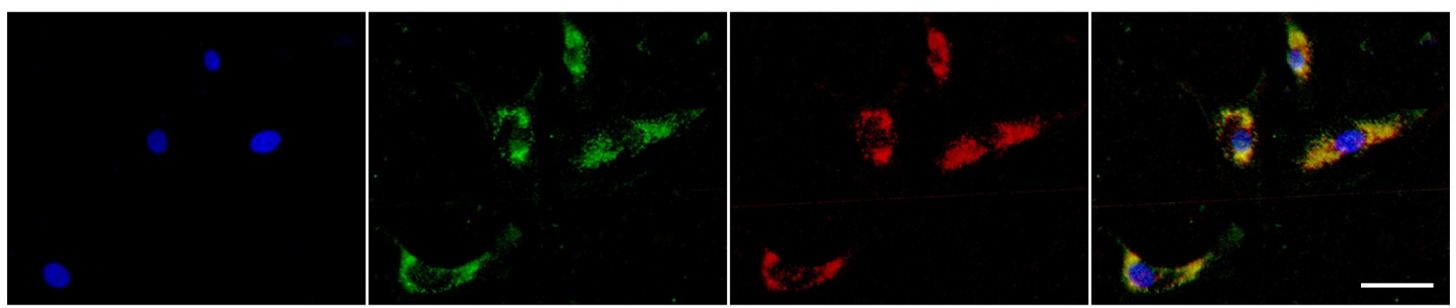

B
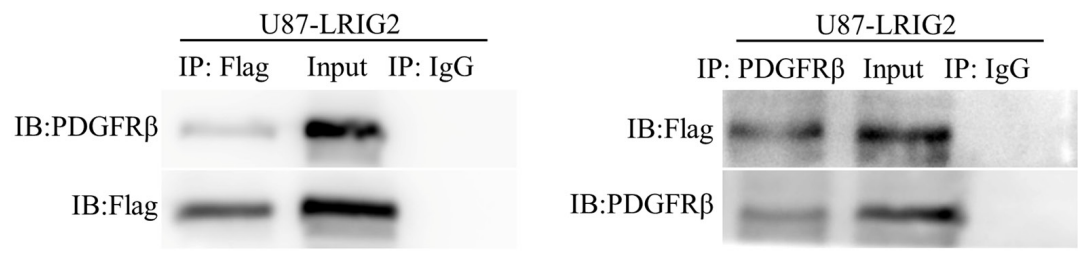

Figure 8. LRIG2 physically interacts with PDGFR $\beta$ in glioblastoma cells. (A) Colocalization of LRIG2 and PDGFR $\beta$ in LRIG2-overexpressing U87 glioblastoma cells. Confocal micrographs reflecting the distribution of fluorescent Flag-LRIG2 (green fluorescence) and PDGFR $\beta$ (red fluorescence) are depicted, along with a merge graph depicting both signals (yellow fluorescence), indicating regions of colocalization (scale bar, $50 \mu \mathrm{m}$ ). (B) Lysates from cells overexpressing LRIG2 were immunoprecipitated with indicated antibodies or corresponding control IgG, and the immunoprecipitates were western blotted with indicated antibodies. Representative western blotting images are shown. The labels of the lanes are indicated as follows: IP, immunoprecipitated with; IB, immunoblotted with; Input, whole cell lysates used as positive controls; IgG, the isotype IgG used as negative control. LRIG2, leucine-rich repeats and immunoglobulin-like domain 2; PDGFR $\beta$, platelet-derived growth factor receptor $\beta$.

A
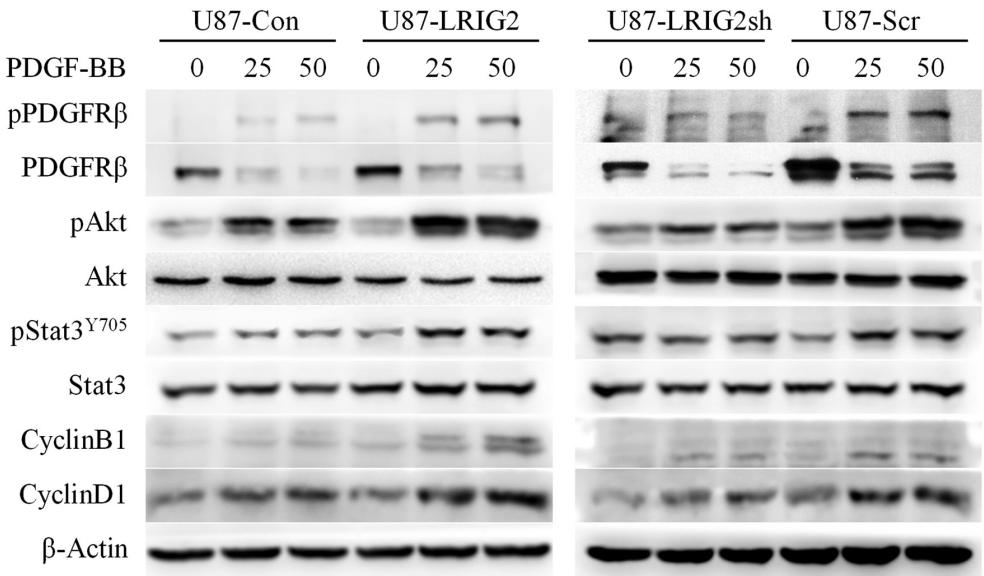

B
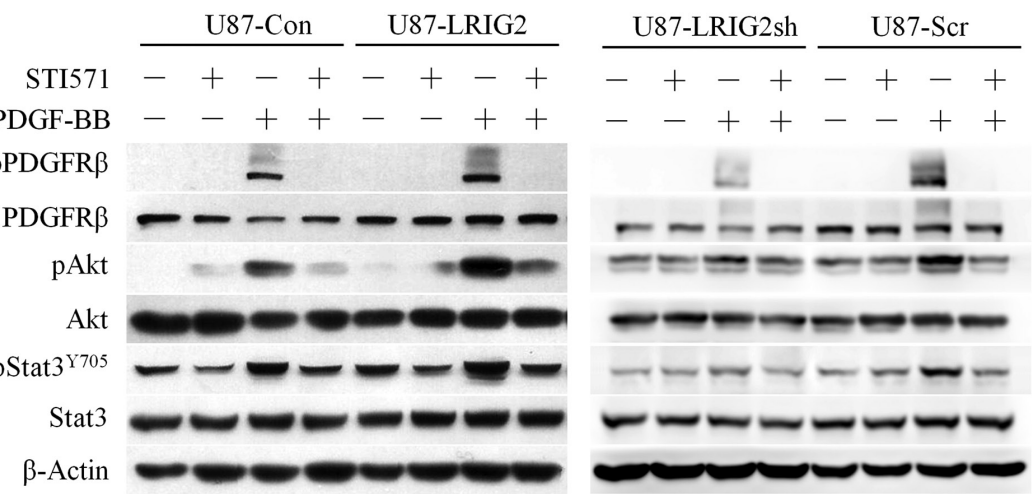

Figure 9. LRIG2 promotes PDGF-BB-induced activation of PDGFR $\beta$ and its downstream Akt and Stat3 signaling pathways. Cells were serum-starved for $24 \mathrm{~h}$ and then treated as follows: (A) With or without indicated concentrations of PDGF-BB (ng/ml) in Dulbecco's modified Eagle's medium with $0.5 \%$ fetal bovine serum for $24 \mathrm{~h}$; or (B) with STI571 $(20 \mu \mathrm{M})$ for $2 \mathrm{~h}$ prior to a 10-min exposure to PDGF-BB $(50 \mathrm{ng} / \mathrm{ml})$. Total cell lysates were subjected to western blot analysis using the indicated antibodies. $\beta$-actin served as control of protein loading. Each panel depicts a representative western blot of three different experiments with similar results. LRIG2, leucine-rich repeats and immunoglobulin-like domain 2; PDGFR $\beta$, platelet-derived growth factor receptor $\beta$.

Akt and Stat3 pathways to promote cell cycle progression and proliferation of GBM cells by modulating PDGFR $\beta$ activation.
However, Rondahl et al (20) reported that Lrig2 exerted no effects on PDGFR protein levels or the phosphorylation events 
of PDGFR and Akt in mouse embryonic fibroblasts (MEFs), which was distinct from the results of the present study. Due to the fact that the experiments by Rondahl et al were carried out in MEFs, rather than in tumor cells or GBM cells, we hypothesized that the positive regulation of LRIG2 on PDGFR $\beta$ and its downstream pathways may be prominent in GBM cells or other tumor cells, but subtle in non-tumor cells, highlighting that the role of LRIG2 in the modulation of PDGFR signaling is oncogenic and tumor-specific.

In addition to the pivotal role of LRIG2 in the positive regulation of PDGFR signaling in GBM, we previously demonstrated that LRIG2 also positively regulated EGFR signaling, another common dysregulated RTK signaling pathway in GBM, to exert its pro-tumor effects on GBM $(21,22)$, indicating that LRIG2 may play a key role in the genesis and progression of GBM by positively regulating EGFR and PDGFR signaling, the most commonly dysregulated RTKs in GBM. Over the past decade, GBM research has primarily focused on determining and investigating specific inhibitors targeting key RTK signaling pathways, in order to develop novel and effective therapeutic strategies to improve the prognosis of GBM. However, accumulating clinical trial data to date, evaluating RTK inhibitors, including EGFR tyrosine kinase inhibitors (TKIs) and PDGFR TKIs, support the conclusion that RTK inhibitors are not beneficial for patients with GBM and the underlying mechanisms have not yet been fully elucidated (33). It has been demonstrated that multiple RTKs may be activated simultaneously in GBM cells $(34,35)$, which is a plausible explanation for the disappointing effects of RTK inhibitors, in that targeting specific RTK signaling may result in compensatory activation of alternative signaling mediators. Our determination of the positive regulation of LRIG2 on EGFR and PDGFR, the most common dysregulated RTKs in GBM, not only improved our understanding of the endogenous regulatory mechanisms of EGFR and PDGFR, but also provided one potential explanation for the limited clinical responses to anti-EGFR or anti-PDGFR therapies using corresponding inhibitors, which is that targeting specific RTK signaling may confer activation of alternative RTK signaling through LRIG2, the key mediator of multiple RTK signals. Our data provide the rationale for targeting LRIG2 as a strategy to achieve the dual inhibition of EGFR and PDGFR signaling in GBM, highlighting the possibility of targeting LRIG2 as a promising therapeutic strategy for the future treatment of GBM.

To the best of our knowledge, this is the first study to demonstrate that LRIG2 positively regulates multiple RTKs, including PDGFR $\beta$, EGFR (22) and other RTKs in GBM (data not published). The role of LRIG2 in the positive regulation of RTKs in glioma is contrary to the role of LRIG1, which has been well documented to negatively regulate multiple RTKs and is a proposed tumor suppressor in a wide spectrum of human cancer types, including glioma $(6,23,24)$. LRIG1 has been demonstrated to restrict RTK signaling through different mechanisms, such as enhancing the degradation of EGFR in a ligand-induced cbl-mediated ubiquitylation process $(7,36)$, destabilizing the MET receptor in a lysosome-dependent and cbl-independent manner (8), and inhibiting glial cell-derived neurotrophic factor binding with RET (9). Little is known regarding the mechanisms through which LRIG2 positively regulates RTKs. In the present study, it was demonstrated that LRIG2 could physically interact with PDGFR $\beta$ in the cytoplasm and increase the PDGF-BB-induced total expression level and phosphorylation of PDGFR $\beta$, which was in line with the effects of LRIG2 on EGFR (22). We hypothesized that the physical interaction of LRIG2 with RTKs may stabilize the expression level of RTKs in GBM cells; however, the critical questions of how LRIG2 stabilizes RTKs, and why the function of LRIG2 as a tumor promoter is largely distinct from that of LRIG1 in glioma, remain unanswered and require further investigation.

Collectively, it was demonstrated that LRIG2 promoted cell cycle progression and proliferation of GBM cells in vitro and in vivo by positively regulating the PDGFR $\beta$ signaling pathways, a critical aberrant oncogenic RTK signaling modulated by LRIG2 in GBM, similar to the previously reported effects on EGFR signaling. Targeting LRIG2, the key oncogenic regulator of multiple RTKs signals, appears to be promising for developing novel therapeutic strategies for the future treatment of GBM.

\section{Acknowledgements}

The authors would like to thank Anding Liu for providing technical assistance.

\section{Funding}

The present study was supported by grants from the National Natural Science Foundation of China (nos. 81702480 and 81472364).

\section{Availability of data and materials}

The analyzed data sets generated during the study are available from the corresponding author on reasonable request.

\section{Authors' contributions}

QX performed the cell biology experiments, is responsible for the animal experiments, and drafted the manuscript. MD and WZ contributed to western blotting experiments. FC and KW collected the fresh human GBM samples. FM performed the immunohistochemistry experiments. HW and RX carried out the statistical analyses. BW, TL and DG designed the experiments and critically revised the manuscript. All the authors provided final approval for the submission.

\section{Ethics approval and consent to participate}

Collection and use of patient tumor tissue samples was approved by the local ethics committees (Institutional Review Board, Tongji Hospital, Tongji Medical College, Huazhong University of Science and Technology, IRB ID: 20121202) and the consents of all the patients were written and approved. All animal experiments were conducted in accordance with the institutional animal research guidelines approved by local Ethics Committee (Institutional Review Board of Experimental Animals, Tongji Hospital, Tongji Medical College, Huazhong University of Science and Technology, IRB ID: 2011A01). 


\section{Patient consent for publication}

Not applicable.

\section{Competing interests}

The authors declare that they have no competing interests.

\section{References}

1. Wen PY and Reardon DA: Neuro-oncology in 2015: Progress in glioma diagnosis, classification and treatment. Nat Rev Neurol 12: 69-70, 2016.

2. Patel M, Vogelbaum MA, Barnett GH, Jalali R and Ahluwalia MS: Molecular targeted therapy in recurrent glioblastoma: Current challenges and future directions. Expert Opin Investig Drugs 21 : 1247-1266, 2012.

3. Cancer Genome Atlas Research Network: Comprehensive genomic characterization defines human glioblastoma genes and core pathways. Nature 455: 1061-1068, 2008.

4. Paulsson J, Lindh MB, Jarvius M, Puputti M, Nistér M, Nupponen NN, Paulus W, Söderberg O, Dresemann G von Deimling A, et al: Prognostic but not predictive role of platelet-derived growth factor receptors in patients with recurrent glioblastoma. Int J Cancer 128: 1981-1988, 2011.

5. Guo D, Holmlund C,Henriksson R and Hedman H: The LRIG gene family has three vertebrate paralogs widely expressed in human and mouse tissues and a homolog in Ascidiacea. Genomics 84: 157-165, 2004

6. Simion C, Cedano-Prieto ME and Sweeney C: The LRIG family Enigmatic regulators of growth factor receptor signaling. Endocr Relat Cancer 21: R431-R443, 2014

7. Gur G, Rubin C, Katz M, Amit I, Citri A, Nilsson J, Amariglio N, Henriksson $\mathrm{R}$, Rechavi $\mathrm{G}$, Hedman $\mathrm{H}$, et al: LRIG1 restricts growth factor signaling by enhancing receptor ubiquitylation and degradation. EMBO J 23: 3270-3281, 2004.

8. Shattuck DL, Miller JK, Laederich M, Funes M, Petersen H, Carraway KL III and Sweeney C: LRIG1 is a novel negative regulator of the Met receptor and opposes Met and Her2 synergy. Mol Cell Biol 27: 1934-1946, 2007.

9. Ledda F, Bieraugel O, Fard SS, Vilar M and Paratcha G: Lrig1 is an endogenous inhibitor of Ret receptor tyrosine kinase activation, downstream signaling, and biological responses to GDNF. J Neurosci 28: 39-49, 2008.

10. Lindström AK, Ekman K, Stendahl U, Tot T, Henriksson R, Hedman H and Hellberg D: LRIG1 and squamous epithelial uterine cervical cancer: Correlation to prognosis, other tumor markers, sex steroid hormones, and smoking. Int J Gynecol Cancer 18: 312-317, 2008.

11. Hellberg D, Tot T and Stendahl U: Pitfalls in immunohistochemical validation of tumor marker expression - exemplified in invasive cancer of the uterine cervix. Gynecol Oncol 112: 235-240, 2009.

12. Krig SR, Frietze S, Simion C, Miller JK, Fry WH, Rafidi H, Kotelawala L, Qi L, Griffith OL, Gray JW, et al: Lrig1 is an estrogen-regulated growth suppressor and correlates with longer relapse-free survival in ERalpha-positive breast cancer. Mol Cancer Res 9: 1406-1417, 2011.

13. Wang Y, Poulin EJ and Coffey RJ: LRIG1 is a triple threat: ERBB negative regulator, intestinal stem cell marker and tumour suppressor. Br J Cancer 108: 1765-1770, 2013.

14. Sheu JJ,Lee CC, Hua CH, Li CI, Lai MT, Lee SC, Cheng J, Chen CM, Chan C, Chao SC, et al: LRIG1 modulates aggressiveness of head and neck cancers by regulating EGFR-MAPK-SPHK1 signaling and extracellular matrix remodeling. Oncogene 33: 1375-1384, 2014.

15. Ranhem C, Lillsunde Larsson G, Hedman H, Lindquist D Karlsson MG, Hellström AC, Östensson E, Sorbe B, Hellman K and Andersson S: Expression of LRIG proteins as possible prognostic factors in primary vaginal carcinoma. PLoS One 12: e0183816, 2017.

16. Rouam S, Moreau T and Broët P: Identifying common prognostic factors in genomic cancer studies: A novel index for censored outcomes. BMC Bioinformatics 11: 150, 2010.

17. Guo D, Nilsson J, Haapasalo H, Raheem O, Bergenheim T, Hedman $\mathrm{H}$ and Henriksson R: Perinuclear leucine-rich repeats and immunoglobulin-like domain proteins (LRIG1-3) as prognostic indicators in astrocytic tumors. Acta Neuropathol 111: 238-246, 2006
18. Holmlund C, Haapasalo H, Yi W, Raheem O, Brännström T, Bragge H, Henriksson R, Hedman H: Cytoplasmic LRIG2 expression is associated with poor oligodendroglioma patient survival. Neuropathology 29: 242-247, 2009.

19. Hedman H, Lindström AK, Tot T, Stendahl U, Henriksson R and Hellberg D: LRIG2 in contrast to LRIG1 predicts poor survival in early-stage squamous cell carcinoma of the uterine cervix. Acta Oncol 49: 812-815, 2010.

20. Rondahl V, Holmlund C, Karlsson T, Wang B, Faraz M, Henriksson R and Hedman H: Lrig2-deficient mice are protected against PDGFB-induced glioma. PLoS One 8: e73635, 2013.

21. Wang B, Han L, Chen R, Cai M, Han F, Lei T and Guo D: Downregulation of LRIG2 expression by RNA interference inhibits glioblastoma cell (GL15) growth, causes cell cycle redistribution, increases cell apoptosis and enhances cell adhesion and invasion in vitro. Cancer Biol Ther 8: 1018-1023, 2009.

22. Xiao Q, Tan Y, Guo Y, Yang H, Mao F, Xie R, Wang B, Lei T and Guo D: Soluble LRIG2 ectodomain is released from glioblastoma cells and promotes the proliferation and inhibits the apoptosis of glioblastoma cells in vitro and in vivo in a similar manner to the full-length LRIG2. PLoS One 9: e111419, 2014.

23. Mao F, Wang B, Xiao Q, Xi G, Sun W, Zhang H, Ye F, Wan F, Guo D, Lei T, et al: A role for LRIG1 in the regulation of malignant glioma aggressiveness. Int J Oncol 42: 1081-1087, 2013.

24. Xie R, Yang H, Xiao Q, Mao F, Zhang S, Ye F, Wan F, Wang B, Lei T and Guo D: Downregulation of LRIG1 expression by RNA interference promotes the aggressive properties of glioma cells via EGFR/Akt/c-Myc activation. Oncol Rep 29: 177-184, 2013.

25. Allen M, Bjerke M, Edlund H, Nelander S and Westermark B: Origin of the U87MG glioma cell line: Good news and bad news. Sci Transl Med 8: 354re3, 2016.

26. Holmlund C, Nilsson J, Guo D, Starefeldt A, Golovleva I, Henriksson $\mathrm{R}$ and Hedman $\mathrm{H}$ : Characterization and tissuespecific expression of human LRIG2. Gene 332: 35-43, 2004.

27. Andrae J, Gallini R and Betsholtz C: Role of platelet-derived growth factors in physiology and medicine. Genes Dev 22: 1276-1312, 2008

28. Nazarenko I, Hede SM, He X, Hedrén A, Thompson J, Lindström MS and Nistér M: PDGF and PDGF receptors in glioma. Ups J Med Sci 117: 99-112, 2012.

29. Nakada M, Kita D, Watanabe T, Hayashi Y, Teng L, Pyko IV and Hamada J: Aberrant signaling pathways in glioma. Cancers (Basel) 3: 3242-3278, 2011.

30. Paradowski M, Bilinska M and Bar J: Characteristics of the expression of KAI1/CD82 and PDGFR $\beta$ and their impact on glioma progression. Folia Neuropathol 54: 241-248, 2016.

31. Holland EC, Celestino J, Dai C, Schaefer L, Sawaya RE and Fuller GN: Combined activation of Ras and Akt in neural progenitors induces glioblastoma formation in mice. Nat Genet 25: 55-57, 2000.

32. Brantley EC and Benveniste EN: Signal transducer and activator of transcription-3: A molecular hub for signaling pathways in gliomas. Mol Cancer Res 6: 675-684, 2008.

33. Reardon DA, Wen PY and Mellinghoff IK: Targeted molecular therapies against epidermal growth factor receptor: past experiences and challenges. Neuro Oncol 16 (Suppl 8): viii7-13, 2014.

34. Van Meir EG, Hadjipanayis CG, Norden AD, Shu HK, Wen PY and Olson JJ: Exciting new advances in neuro-oncology: The avenue to a cure for malignant glioma. CA Cancer J Clin 60: 166-193, 2010.

35. Snuderl M, Fazlollahi L, Le LP, Nitta M, Zhelyazkova BH, Davidson CJ, Akhavanfard S, Cahill DP, Aldape KD, Betensky RA, et al: Mosaic amplification of multiple receptor tyrosine kinase genes in glioblastoma. Cancer Cell 20: 810-817, 2011.

36. Laederich MB, Funes-Duran M, Yen L, Ingalla E, Wu X, Carraway KL III and Sweeney C: The leucine-rich repeat protein LRIG1 is a negative regulator of ErbB family receptor tyrosine kinases. J Biol Chem 279: 47050-47056, 2004.

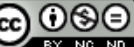

This work is licensed under a Creative Commons Attribution-NonCommercial-NoDerivatives 4.0 International (CC BY-NC-ND 4.0) License. 